\title{
La comisión de crímenes contra la humanidad por empresas transnacionales y la ampliación de la competencia ratione personae de la Corte Penal Internacional
}

\author{
The commission of crimes against humanity by transnational \\ corporations and the extension of the jurisdiction ratione \\ personae of the International Criminal Court
}

\author{
Libia Arenal Lora \\ Universidad de Sevilla \\ marenal@us.es
}

Cómo citar/Citation: Arenal, Libia. 2020. «La comisión de crímenes contra la humanidad por empresas transnacionales y la ampliación de la competencia ratione personae de la Corte Penal Internacional». Deusto Journal of Human Rights, No. 6: 13-41. doi: http://dx.doi.org/10.18543/djhr.1872.

\begin{abstract}
Sumario: Introducción. 1. Las empresas transnacionales y el concepto de «organización» en el artículo 7 del Estatuto de Roma. Análisis del «test de capacidad» en la jurisprudencia de la Corte Penal Internacional. 2. La competencia de la Corte Penal Internacional y su extensión ratione personae a las empresas transnacionales. Conclusiones. Referencias bibliográficas.
\end{abstract}

Resumen: ¿Pueden las empresas transnacionales perpetrar crímenes contra la humanidad y ser investigadas y procesadas por ello en virtud de las normas e instituciones propias del Derecho internacional penal (DIP)? Este trabajo pretende ofrecer una respuesta a esta pregunta analizando las posibilidades y obstáculos jurídicos que pueden encontrarse en la aplicación del Estatuto de Roma de la Corte Penal Internacional (CPI). En primer lugar, se examina si las empresas transnacionales pueden ser entidades susceptibles de integrarse en el concepto «organización» del art. 7 del Estatuto de Roma de la CPI, convertirse en un actor detrás del elemento de la política en la comisión de los crímenes contra la humanidad y ser penalmente responsable por estos comportamientos en Derecho internacional. Para dar respuesta a esta cuestión se analiza la jurisprudencia reciente de la CPI. En segundo lugar, se aborda la posible extensión de la competencia ratione personae de la CPI a las personas jurídicas, en este caso a las empresas transnacionales, teniendo en 
consideración argumentos presentes en el Derecho internacional público, en el sistema de la CPI y en el Derecho penal

Palabras clave: crímenes contra la humanidad, responsabilidad penal internacional de las empresas, competencia ratione personae de la Corte Penal Internacional.

Abstract: Can transnational corporations commit crimes against humanity and be investigated and prosecuted by the International Criminal Court (ICC)? This article intends to provide an answer to this question by analyzing the legal possibilities and obstacles that could be found in the application of the Rome Statute of the ICC. First, the article examines whether corporations could meet the requirements established by the recent jurisprudence of the ICC in order to be considered as the concept of "organization» stated in art. 7 of the Rome Statute (RS), complying with the political element of crimes against humanity, and being criminally responsible for these crimes in international law. Secondly, it addresses the extension of the jurisdiction ratione personae of the ICC to include the legal person, specifically corporations, taking into consideration the legal arguments emanating from international law, the ICC system and criminal law.

Keywords: crimes against humanity, international criminal corporate responsibility, jurisdiction ratione personae of the ICC. 


\section{Introducción ${ }^{1}$}

Las empresas transnacionales ${ }^{2}$, a través de las actividades y operaciones que desarrollan en los diversos sectores económicos en los que actúan, han contribuido de manera significativa a la comisión de crímenes internacionales, y ésta es una cuestión que a día de hoy no se pone en duda.

Tras la Segunda Guerra Mundial, los procesos que se celebraron por el Tribunal Penal Internacional Militar de Núremberg, constituido por la Carta de Londres de 1945, contra los industriales alemanes Flick, Krup y Farben ilustraron con enorme claridad la colaboración de las empresas alemanas con el régimen nacionalsocialista en la comisión de los execrables crímenes contra la humanidad y crímenes de guerra que conmocionaron a toda la comunidad internacional. Estos casos fueron pioneros en la batalla de exigir responsabilidad a las empresas por la comisión de crímenes internacionales. Los juicios concluyeron con condenas dictadas contra importantes hombres de la industria alemana, ligados al régimen Nazi, por complicidad en estos atroces crímenes, poniéndose de manifiesto en las acusaciones la instrumentalidad de las empresas en la comisión de aquellos actos de barbarie ${ }^{3}$.

Desde entonces hasta la actualidad, ningún otro tribunal penal internacional ha condenado a un empresario, menos aún a la persona jurídica que es la empresa, por la comisión de crímenes internacionales. Solo el Tribunal Especial para el Líbano (TEL), creado por la resolución 1757 del Consejo de Seguridad de la ONU, de 30 de mayo de 2007, para juzgar los actos terroristas relacionados con el asesinato de Rafik Hariri, parece una excepción en este sentido. El TEL confirmó la existencia de responsabilidad de las empresas en Derecho internacional en el caso conocido como The Al-Jadeed ${ }^{4}$. Sin embargo, la competencia

1 Este artículo se enmarca en el Proyecto de investigación Gobernanza y Aplicación de la Responsabilidad Social Empresarial en la Unión Europea (DER2017-85834-R), financiado por el Ministerio de Economía y Competitividad.

2 El interés que despiertan las empresas transnacionales de cara a este trabajo se fundamenta en el hecho de ser entidades que ostentan un enorme poder económico y financiero, que despliegan su actividad globalmente a través de una compleja estructura de filiales y subsidiarias, siendo estas circunstancias lo que les permiten evitar el cumplimiento de normas y escapar al control judicial de los países en los que operan o en los que tienen sus matrices, dejando un saldo de impunidad cuando se ven implicadas en violaciones graves de derechos humanos o en la comisión de crímenes internacionales.

3 The United States of America v. Carl Krauch, et al.

4 Special Tribunal for Lebanon (STL), Al Jadeed S.A.L. \& Ms Khayat (STL-14-05). 
del Tribunal sobre la empresa Al Jadeed S.A.L. se atribuyó en virtud del art. 60 bis de las Reglas de Procedimiento y Pruebas, bajo una acusación de obstrucción a la justicia ${ }^{5}$. La extensión de la jurisdicción ratione personae del Tribunal se produjo, en todo caso, con el objeto de asegurar que el desarrollo del proceso judicial se realizara con garantías plenas. Por consiguiente, no puede decirse que éste sea un caso estrictamente de extensión de la competencia de un tribunal internacional sobre las empresas por su participación en crímenes internacionales ${ }^{6}$.

A pesar de este vacío en la práctica de los tribunales internacionales, una tendencia distinta se ha visto en los foros judiciales nacionales ante los que han sido llevadas empresas, en particular empresas transnacionales, exigiendo su responsabilidad por su implicación en la comisión de crímenes internacionales, recordándonos que estas aberrantes conductas no son hechos aislados, sino una práctica más habitual de lo que resulta en apariencia.

Algunos de los más importantes y recientes casos que pueden enunciarse son el de las empresas Titan Corporation y CACl International INC., acusadas de torturas y otros tratos crueles e inhumanos perpetrados contra prisioneros en la cárcel de Abu Ghraib en Iraq $^{7}$ que, aunque terminó con un acuerdo extrajudicial, demostró la responsabilidad de la empresa por los hechos ocurridos y es el primer caso en el que una empresa militar y de seguridad privada acepta su responsabilidad en estos crímenes. La empresa cementera europea Lafarge ha sido imputada antes los tribunales franceses por complicidad en crímenes contra la humanidad, financiación de grupos terroristas y violación de seguridad de los trabajadores en Siria ${ }^{8}$. En Holanda, en

5 Case no. STL-14-05/PT/CJ/F0054/20140724/R001208-R001242/EN/dm. Decision of Contempt Judge, 24. Julio 2014. paras. 29-30.

6 Hay que tener en cuenta que el Estatuto del TEL señala que sus disposiciones serán interpretadas de acuerdo al espíritu del propio Estatuto y en orden de preferencia: CVDT de 1969, estándares de derechos humanos, los principios generales del Derecho internacional penal y el Código procesal penal libanes bajo el que las personas jurídicas sí podrían ser responsables por el comportamiento que se les atribuye en el caso. Rule 3 (A) STL RPE.

7 Una sinopsis de estos casos puede verse la página web ECCR; para Saleh, et al. v. Titan, et al. en https://ccrjustice.org/home/what-we-do/our-cases/saleh-et-al-v-titan-et-al; para Al Shimari v. CACl en https://ccrjustice.org/home/what-we-do/our-cases/al-shimariv-caci-et-al; Al-Quraishi, et al. v. Nakhla and L-3 Services ((formerly Titan Corporation, now Engility) en https://ccrjustice.org/home/what-we-do/our-cases/al-quraishi-et-al-vnakhla-and-I-3-services

8 Una sinopsis de este caso puede verse en: https://www.ecchr.eu/en/case/madein-europe-bombed-in-yemen. El 7 de noviembre de 2019 la Sala de instrucción del 


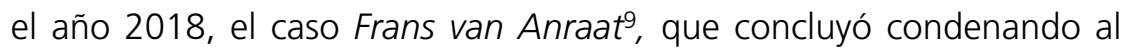
empresario por complicidad en crímenes de guerra por suministrar al gobierno iraquí químicos necesarios para la producción de gas mostaza utilizado en las masacres contra la minoría Kurda en Iraq. También el caso contra Guus Kouwenhoven ${ }^{10}$, dueño de una de las grandes empresas madereras en Europa, que finalizó en los tribunales de la Haya en 2017 con una condena por tráfico ilícito de armas y por complicidad en crímenes de guerra durante el conflicto bélico en Liberia. Finalmente, el emblemático caso de la Ford en Argentina, que concluyó en 2019 con la condena de algunos directivos de la empresa transnacional no solo por ser cómplices de la dictadura cívico-militar, sino también como partícipes necesarios de los crímenes del terrorismo de Estado ${ }^{11}$.

En todos estos casos presentados ante foros judiciales nacionales ha quedado establecida algún tipo de responsabilidad penal o civil de los empresarios por su complicidad o cooperación a través de la llamada «empresa criminal conjunta» en la comisión de los crímenes internacionales, y han mostrado los escenarios comunes en los que las empresas se ven implicadas en crímenes cometidos por terceros actores: apoyo a regímenes militares y dictatoriales a través de diversas actividades de tipo colaborativo de las que obtienen un beneficio directo; fomento o contribución al recrudecimiento de las acciones violentas en zonas donde existen guerras o conflictos armados para asegurar el desarrollo de su actividad económica apoyando o financiado a algunas de las partes del conflicto; y, cooperación con la realización de estos crímenes, mediante la provisión de servicios militares y de inteligencia (Kaleck y Saage-MaaB 2010).

Sin embargo, aunque estas decisiones judiciales suponen un importante avance en la búsqueda de fijar la responsabilidad de las empresas por su participación en estos aberrantes crímenes, no parecen suficientes ni para dimensionar la gravedad de la naturaleza

Tribunal de Apelación de París anuló la acusación por complicidad en crímenes contra la humanidad, pero mantuvo el cargo de financiación del terrorismo. Véase: https://www. ecchr.eu/nc/en/press-release/sherpa-and-ecchr-to-appeal-decision-in-lafargesyria-caseat-french-supreme-court/

9 The Public Prosecutor v. Frans Cornelis Adrianus van Anraat, District Court of The Hague, The Netherlands (Case n. ${ }^{\circ}$ 09/751003-04), Sentence, 23 December 2005.

10 The Public Prosecutor v. Guus Kouwenhoven, Court of Appeal of The Hague, The Netherlands, (Case n. ${ }^{\circ}$ 220043306) (ECLI: NL: GHSGR: 2008: BC6068), Judgment, 21 April 2017.

11 Poder Judicial de la Nación de Argentina, año 2019, Sentencia Causas n. ${ }^{\circ} 2855$ y 2358. 
y de las consecuencias de una forma de participación de las empresas transnacionales más directa y autónoma de terceros actores, ni para exigir su responsabilidad en calidad de personas jurídicas de forma complementaria a la responsabilidad de los individuos que actúan en su nombre y representación. La ausencia de normas y de mecanismos adecuados para perseguir y exigir a las empresas responsabilidad por sus crímenes produce insuficiente preventiva, dejan sus estructuras intactas perpetuando sus comportamientos y fomentan la impunidad.

El DIP, sector del ordenamiento jurídico internacional en el que tiene origen la tipificación de los crímenes internacionales y la consagración del principio de responsabilidad internacional del individuo, no ha ofrecido a día de hoy respuestas a dos preocupaciones fundamentales sobre esta cuestión: ¿pueden las empresas transnacionales ser responsables de la comisión de crímenes contra la humanidad, más allá del hecho de estar involucrados en los actos que forman parte del ataque, cuando éstos se llevan a cabo de acuerdo a una política corporativa en virtud del art. 7 del Estatuto de Roma? Y, ¿es posible ampliar la competencia ratione personae de la CPI sobre las empresas transnacionales y atribuirle responsabilidad penal a la organización por la comisión de esta categoría de crímenes?

Para dar respuesta a estas preguntas se analizará el concepto de organización incluida en el art. 7.2 del Estatuto de Roma, sobre el que arroja luz el «test de capacidad» formulado por la jurisprudencia de la $\mathrm{CPI}$ con relación al caso sobre la violencia postelectoral que tuvo lugar en Kenia en el año $2017^{12}$ y que ha sido seguido posteriormente en otras causas como la de Routo y Katanga ${ }^{13}$. Una vez esto, se abordará la responsabilidad de las empresas transnacionales por la comisión de crímenes contra la humanidad de acuerdo al Estatuto de Roma, cuestión no resuelta por el DIP. Finalmente, se analizará la posible jurisdicción de la CPI sobre las actividades de las empresas que puedan ser calificadas como crímenes contra la humanidad y, de manera particular, la posible ampliación de competencia ratione materiae de la $\mathrm{CPI}$ sobre la persona jurídica.

12 Case n. ICC-01 / 09-19-Corr-tFRA); Case n. ICC-01/09-02/11) [de aquí en adelante causa Kenyatta 2011]; Case n. ICC-01/09-02/11) [de aquí en adelante causa Kenyatta 2012].

13 Case n. ICC-01/09-01/11-373 [de aquí en adelante, causa Ruto 2012]; Case No. ICC-01/04-01/07, Judgment pursuant to Article 74 of the Statute, 7 March 2014, Trial Chamber II, International Criminal Court. 


\section{Las empresas transnacionales y el concepto de "organización» en el art. 7 del Estatuto de Roma. Análisis del "test de capacidad» en la jurisprudencia de la Corte Penal Internacional}

La prohibición de cometer crímenes contra la humanidad ha tenido como objetivo fundamental el control del abuso del poder del Estado ${ }^{14}$ contra la población civil a través de la comisión de actos considerados aberrantes e inhumanos (Bassiouni 2011). Después del largo recorrido normativo de los crímenes contra la humanidad, desde la adopción del Estatuto de Núremberg, impulsado por los trabajos de Comisión de Derecho Internacional (CDI), y la notable influencia de la jurisprudencia de los Tribunales Penales Internacionales ad hoc (Márquez 2008a), esta categoría de crímenes ha sido tipificada en el art. 7 del Estatuto de Roma como una serie de actos inhumanos que tendrán lugar cuando se cometan «como parte de un ataque generalizado o sistemático contra una población civil y con conocimiento de dicho ataque», siendo que se entenderá por ataque contra una población civil «una línea de conducta que implique la comisión múltiple de actos mencionados (...) de conformidad con la política de un Estado o de una organización de cometer ese ataque o para promover esa política» ${ }^{15}$.

De la redacción de este artículo, llaman la atención dos elementos a los efectos de este trabajo. Por un lado, el Estatuto de la CPI es el primer instrumento jurídico internacional que incluye el requisito de «la política del Estado o de la organización» en la definición de los crímenes contra la humanidad, lo que refleja ese desarrollo normativo de los crímenes desde Núremberg (McAuliffe de Guzman 2000, CDI 2019). El elemento de la política, la dirección o la instigación por parte de un Gobierno o una organización o grupo es la característica esencial de los crímenes contra la humanidad, lo que los diferencia de los crímenes ordinarios (Márquez 2008b). Por otro lado, establece que la política de cometer los crímenes ha de ser la de un Estado o de una "organización» de cometer el ataque contra la población o de promover esa política.

Si bien la jurisprudencia de los Tribunales Penales Internacionales ad hoc se ha pronunciado en varias ocasiones sobre el hecho de que el elemento de la política podía ser satisfecho por una organización

14 Cuando en Núremberg se citó "crimes are committed by men, not by abstract entities», el término "entidades abstractas» era una referencia directa al Estado.

15 ONU. Asamblea General, Estatuto de Roma de la Corte Penal Internacional, 17 Julio 1998, Art. 7 (UN. Treaty Series, vol. 2187, n. 38544). 
no estrictamente estatal ${ }^{16}$ - gobiernos regionales o locales y grupos armados con características estatales - y la doctrina científica ha evitado interpretar la extensión de la calificación jurídica de los crímenes contra la humanidad cometidos de manera generalizada o sistemática por asesinos en serie, mafias o bandas terroristas (Bassiouni 2011 y Schabas 2008b), la aplicación del Estatuto de Roma podría aportar un nuevo desarrollo para el estudio de los crímenes contra la humanidad y la participación en ellos de actores privados.

En este sentido, aunque la expresión «organización» en el Estatuto de Roma parezca una reminiscencia del término "organización criminal», que fue empleado en los juicios de Núremberg para tratar la criminalidad masiva, organizada, voluntaria e intencionada del Gabinete del Gobierno Nazi, la Alta Dirección del Partido Nazi, las SS, la Gestapo, la SA y el Estado Mayor y Oficiales Superiores de las Fuerzas Armadas Alemanas (Fraser 2017 y Jørgensen 2001), y pretenda reflejar la inclusión en su ámbito de aplicación de grupos que se encuentren bajo el control y la influencia del Estado o de organizaciones con características estatales, también es posible plantear un significado más amplio de este término, que alcance a personas jurídicas que tiene como objetivo obtener beneficios privados (UN 1998), como son las empresas transnacionales.

Esta posible ampliación de la noción del término organización puede encontrarse en la jurisprudencia de la CPI, en la medida que ni el Estatuto de Roma, ni el documento de los elementos de los crímenes han precisado esta expresión, mostrando una posición más flexible, o de cierta apertura con relación a su definición en el ámbito del Estatuto y su posible ampliación a diferentes tipos de estructuras organizativas (Di Fillipo 2008, Robinson 2011, Sadat 2013).

La Sala de Cuestiones Preliminares II de la CPI en la Decisión sobre la autorización de la investigación sobre la situación post-electoral en Kenia de 2010 abordó particularmente esta cuestión de cómo debe ser interpretado el término organización en la definición de los crímenes contra la humanidad y enunció los criterios que deben concurrir para que una entidad o grupo quedara incluido dentro del concepto de organización del art. 7 del Estatuto de Roma. Estos criterios han sido posteriormente confirmados en la Decisión de

16 Prosecutor v. Tihomir Blaškić, Case n. IT-95-14-T, Judgment, 3 March 2000, Trial Chamber, International Criminal Tribunal for the Former Yugoslavia, Judicial Reports 2000; Prosecutor v. Fatmir Limaj, Haradin Bala and Isak Musliu, Case n. IT-03-66-T, Judgment, 30 November 2005, Trial Chamber II, International Criminal Tribunal for Former Yugoslavia, 
Petición de comparecencia para Francis Kirimi Muthaura, Uhuru Muigai Kenyatta and Mohammed Hussein Ali de 2011 y en la Decisión de confirmación de cargos contra Kenyatta, Muthara y Hussein Ali y Decisión de confirmación de cargos contra Ruto y otros en de 2012.

En la Decisión sobre la autorización de la investigación sobre la situación post-electoral en Kenia de 2010, la mayoría de la Sala confirmó que el elemento decisivo para la definición de una organización en el sentido del art. 7 del Estatuto de Roma no era la naturaleza formal del grupo, sino que éste tuviera la capacidad de llevar a cabo actos que infringieran valores humanos básicos, proponiendo una serie de criterios o factores no exhaustivos que coadyuvarían a determinar dicha capacidad de actuar ${ }^{17}$.

Estos criterios establecidos por las salas de la CPI, pueden resumirse en los siguientes: estar bajo órdenes de un mando responsable, que el grupo poseyera los medios para llevar a cabo un ataque generalizado o sistemático contra la población civil, que el grupo ejerciera control sobre el territorio, que tuviera como objeto principal llevar a cabo actos criminales contra la población civil, o que hubiera articulado la intención de cometer el ataque ${ }^{18}$.

El análisis que ha realizado la doctrina de estos criterios ha llevado a algunos autores a concluir que la mayoría de la Sala II en la Decisión sobre la situación pos-electoral en Kenia no excluyó la posibilidad de que grupos privados pudieran estar involucrados en crímenes contra la humanidad (Van der Wilt 2014, 307), encontrando esta opinión apoyo en el comentario 5 al art. 21 del Proyecto de Código de crímenes contra la paz y la seguridad de la humanidad de la CDI de 1991, que hacía referencia a "particulares dotados de un poder de hecho y organizados en bandas o grupos criminales», cuyos actos pudieran entrar dentro del ámbito de aplicación del Proyecto ${ }^{19}$.

17 Situation in the Republic of Kenya, Decision, 31 March 2010, para. 90. Posición adoptada también en el caso Katanga. Hay que tener en cuenta las opiniones disidentes del Juez Hans-Peter Kaul —Dissenting Opinion of Judge Hans-Peter Kaul, 31 March 2010 y Dissenting Opinion by Judge Han-Peter 23 January 2011-, en las que señalaba que la definición más amplia o flexible del término organización convertiría los crímenes contra la humanidad en un término utilizado para abordar todo tipo de atrocidades masivas que no fueran estrictamente actos aislados o particulares de violencia.

18 Causa Kenyatta de 2010, párr. 93; causa Kenyatta de 2012, párr. 185.

19 Comisión de Derecho Internacional (CDI). 1991. Anuario de la Comisión de Derecho Internacional. Vol. II, Parte 2. Informe de la Comisión a la Asamblea General sobre la labor realizada en su cuadragésimo tercer período de sesiones. UN Doc. AV CN.4/SER. A/199 I/Add. 1 (Part 2), p. 111. 
Para conocer si las empresas transnacionales son entidades susceptibles de integrarse en el concepto de organización del art. 7 del Estatuto de Roma y, en consecuencia, ser un actor detrás de la política para cometer crímenes contra la humanidad, ha de llevarse a cabo la tarea de analizar sus conductas criminales a la luz del «test de capacidad». Ahora bien, para iniciar este examen relativo a la capacidad de las empresas de infringir valores humanos básicos, se ha de partir desde la premisa que la actividad criminal de las empresas no solo se encuentra asociada a crímenes que tienen una dimensión estrictamente económica -entendiendo como tales los delitos contra el patrimonio y el orden socioeconómico, como son la evasión fiscal, el lavado de dinero, la apropiación indebida, la administración desleal, la bancarrota fraudulenta, la corrupción, soborno de funcionarios públicos, entre otros, los llamados delitos de «cuello blanco»- Esto es así porque, como se describirá a continuación, las actividades y las operaciones de las empresas pueden adentrarse en la comisión de crímenes clásicos que, en el marco de un contexto adecuado, podrían calificarse o ser constitutivos de crímenes contra la humanidad (Smith 2009).

Este tipo de actividades criminales de las empresas a las que nos referimos pueden manifestarse cuando conductas que forman parte de la cadena de desarrollo de la actividad corporativa coinciden o se solapan con alguno de los tipos penales recogidos en el art. 7 del Estatuto de Roma. En este sentido, las prácticas de esclavitud moderna y la trata de personas podrían sobreponerse con el crimen de esclavitud (art. 7.c del Estatuto de Roma) (Oboka 2005, Moran 2014, Van der Wilt 2014). Algunos conocidos casos judiciales confirman que es posible esta relación, destacando entre ellos el que ha involucrado a la multinacional Nestlé, demandada bajo la Ley de Transparencia de las Cadenas de Suministro de California de 2010 ( «SB 657») por el uso de trabajo esclavo en la cadena de producción del pescado de su comida para gatos en el sudeste asiático ${ }^{20}$, así como por prácticas de esclavitud y trabajo infantil en la cadena de producción de cacao desde Costa de Marfil, éste último junto a la multinacional Mars ${ }^{21}$. En estos casos, con independencia del foro judicial o del ámbito de responsabilidad bajo el que se juzgue la comisión de los crímenes, se destaca que la realización de los actos criminales constituyó un medio objetivo para la consecución de los fines que le son propios como

20 Barber v. Nestle USA Case n. SACV 15-01364-CJC (AGRx).

21 Robert Hodson, v. MARS, INC., et al. Case n. 15-cv-04450-RS. 
empresa, la obtención de un beneficio privado, y que se llevaron a cabo «intencionalmente» o «a sabiendas» de su resultado 22 .

Ahora bien, las empresas transnacionales también pueden llevar a cabo actividades criminales ligadas a la realización de los actos tipificados en el art. 7 del Estatuto de Roma cuando la comisión de asesinatos, desplazamientos forzados, o actos de violencia sexual están en conexión con el desarrollo de sus actividades económicas habituales, buscando con ello ganar y afianzar su poder económico, el control social en las áreas en las que operan o maximizar sus beneficios. Los casos de asesinatos de líderes ambientales que pretenden proteger el territorio y los recursos naturales del desarrollo de grandes proyectos extractivos o de mega infraestructuras - por ejemplo, el asesinato de Berta Cáceres, en el que a pesar de ser declaradas culpables siete personas vinculadas con la Empresa DESA y con el ejército hondureño, quedó claro que "esta condena representa solo el escalón más bajo de la estructura criminal»23 - o los de desplazamientos de población, daños a la integridad y la salud física y mental de miles de personas afectadas por la contaminación masiva derivada de la gestión de proyectos de hidrocarburos - por ejemplo, la provocada en la Amazonia ecuatoriana por la multinacional Chevron, ahora Texacoponen de manifiesto la capacidad de las empresas transnacionales para infringir aquellos valores humanos básicos a los que hace referencia la CPI en su jurisprudencia, y cuya protección frente a ataques generalizados o sistemáticos se encuentra en el centro de la prohibición de cometer crímenes contra la humanidad.

Sin embargo, no puede afirmarse que todas las empresas transnacionales, en todas las circunstancias o contextos, tengan la capacidad de vulnerar estos valores fundamentales. Así, la satisfacción de este criterio primigenio debe interpretarse tomando en consideración los otros elementos enumerados por la jurisprudencia de la Corte, los relativos a las características particulares de las organizaciones o grupos, sin que estos supongan en sí mismos una rígida definición

22 La jurisprudencia de los TPI ad hoc ha desarrollado la doctrina del «enfoque orientado al riesgo», por cuanto el conocimiento también incluiría «la conducta de una persona que asume un riesgo premeditado con la esperanza de que éste no cause daños». Prosecutor v. Kunarac et al. (Case n. IT-96-23\& IT-96-23/1-A), Appeals Chamber, Judgment, 12 June 2002 y Prosecutor v. Blaskic (Case n. IT-95-14-T), Trial Chamber, Judgment, 3 March 2000.

${ }^{23}$ Comunicado de la Familia de Berta, el COPINH, Gustavo Castro y los equipos legales de la causa ante el fallo que juzga a Sergio Rodríguez, Mariano Díaz, Douglas Bustillo, Elvin Heriberto Rápalo, Edilson Duarte, Oscar Haroldo Torres y Emerson Duarte. Véase: https://copinh.org/2018/11/comunicado-ante-el-fallo/ 
legal, ni se exija que todos estos elementos hayan de ser totalmente satisfechos 24 .

En primer lugar, el criterio de encontrarse el grupo o la empresa bajo un mando responsable o dominado por una estructura jerárquica, se satisfaría teniendo en consideración la definición del término de empresa transnacional como «una empresa mercantil con ánimo de lucro que se apoya en la expansión internacional de sus actividades empresariales, y por lo tanto a una pluralidad de Estados, para obtener el máximo beneficio posible a partir de una estrategia pensada en términos mundiales, bajo una unidad de gestión centralizada en la empresa matriz, desde la cual se dirigen las actividades transnacionales y se ordena el conjunto de la actividad empresarial» (Bonet, 2013). Lo relevante de este criterio relativo al nivel de organización del grupo per se, desde la perspectiva de los crímenes contra la humanidad, se manifiesta en el hecho de que este grupo cuente con una política o cultura corporativa dentro de la que se llevan a cabo las prácticas o las conductas criminales. Sin este elemento los crímenes a los que nos referimos no se hubieran producido a gran escala, ni de forma generalizada o sistemática, que es lo que caracteriza el umbral de gravedad necesario para distinguirlos de los crímenes ordinarios. La idea es que el grupo se encuentre suficientemente organizado para ser tratado como una entidad distinta a sus miembros bajo el Derecho internacional (Bartels y Fortin 2016) ${ }^{25}$.

En segundo lugar, las empresas transnacionales podrían tener los medios para llevar a cabo un ataque generalizado o sistemático contra la población civil. La CPI hace referencia a la capacidad económica y material para emprender acciones que supongan actos de naturaleza violenta contra la población y, como es sabido, uno de los rasgos que define a las grandes empresas transnacionales de hoy es el del valor de su riqueza ${ }^{26}$, lo que llamamos su soberanía económica, lo que permite que desplieguen un enorme poder que además influye en las decisiones políticas, en la gestación de leyes y en el día a día de los ciudadanos, escapando además en muchas

24 Causa Kenyatta de 2010, párr.93.

25 Se refieren a los grupos armados.

26 De acuerdo a un estudio presentado por Global Justice Now, 69 de las 100 economías más importantes del mundo son empresas y no países. Empresas transnacionales como Walmart, Apple o Shell son más ricas que Rusia, Bélgica o Suiza. Empresas como Facebook o Apple tiene una capitalización en bolsa por un valor que oscila entre los 500.000 y los 850.000 millones de dólares. Véase https://www. globaljustice.org.uk/news/2016/sep/12/10-biggest-corporations-make-more-moneymost-countries-world-combined 
circunstancias de la supervisión y del control de los Estados en los que operan (Naucke 2015)27.

En tercer lugar, aunque las empresas transnacionales no ejerzan propiamente control sobre una parte del territorio - resultaría de una enorme complejidad probar que una empresa ejerce un control estricto sobre el territorio y la población de un Estado soberano-, la interpretación de este elemento podría discurrir más bien en la línea de explicar el control que las empresas ejercen sobre los Estados, neutralizando sus decisiones políticas o capturándolo a través de la corrupción, dejando con ello un interrogante sobre quién es la máxima autoridad. La correlación de fuerzas entre Estados y empresas, al punto de que pudiera explicar los extraordinarios niveles de control de facto logrados por éstas, se explica fundamentalmente en los contextos de los Estados débiles, que vendrían a caracterizarse por una cierta debilidad del imperio de la ley (Donigan 2000), o la debilidad de la cultura de la legalidad (Pérez Caballero 2015, 140). Sin embargo, es manifiesto que esta problemática no solo es propia de países en desarrollo, también ocurre en países más avanzados que se entiende gozan de un sistema democrático más consolidado y de control sobre sus instituciones.

Finalmente, el criterio expuesto por la CPI de tener la organización como objetivo principal de sus actividades criminales atacar a la población civil es el más difícil de establecer respecto de las empresas transnacionales. El fin último de una empresa es obtener ganancias privadas con el ejercicio de su actividad económica y no puede sostenerse que sea el propósito de este tipo de organizaciones generar un daño intencionado sobre la población de manera generalizada o sistemática (Pérez Caballero 2015, 152). Ahora bien, el ataque contra la población civil puede producirse, como ya hemos comentado, cuando alguno de los crímenes comprendidos en el art. 7 del Estatuto de Roma se solapa, forma parte o es el resultado del desarrollo de una política de la empresa, sin la cual los actos no se hubieran producido - casos de esclavitud moderna vinculada a las cadenas productivas, los desplazamientos forzados conectados por proyectos extractivos, los graves daños a la salud física de las personas vulnerables que son utilizadas para realizar pruebas de ensayos de fármacos- Hay que tener en cuenta además con relación a la definición de ataque que éste no solo se construye sobre la idea de la producción de actos de

27 Naucke no hace referencia particularmente a empresas transnacionales, pero sí a otras fuerzas económicas y del mercado, por lo que tomamos como referencia su tesis para aplicarla a las empresas. 
violencia física dirigidos directamente contra la población ${ }^{28}$, ni tiene porqué ser definido de manera restrictiva y circular (Alija 2011). La población civil, en estos casos, tampoco es una víctima incidental29, sino que es consustancial a la posición que ocupa en el contexto en el que se cometen los crímenes de las empresas, asumiéndose la realización del ataque como un riesgo inherente al desarrollo de la política corporativa.

Esta definición más abierta o flexible del término «organización», a la que puede llegarse a través de la interpretación del «test de capacidad» de la CPI, es apoyada por una parte de la doctrina. Di Filippo (2008) ha señalado, por ejemplo, que los criterios expuestos por la CPI pueden ser satisfechos por grupos privados y que no hay razón para distinguir la gravedad de las conductas en virtud de los elementos que definen al grupo per se. Robinson (2011) sugiere que entidades organizadas que dirijan, instigan o promuevan la comisión de graves crímenes, calificarían como una organización bajo el Estatuto de la CPI. En esta misma línea, Sadat (2013) ha precavido que una construcción limitada del término organización del art. 7.2 a las organizaciones con características estatales ignoraría el desarrollo del DIP desde Núremberg.

Asimismo, la CDI (2019), al elaborar el borrador de Proyecto de artículos sobre la prevención y el castigo de los crímenes contra la humanidad, adoptado en su 71. a sesión en 201930, también ha recuperado la jurisprudencia de la CIP para abordar en análisis del concepto "organización» en los crímenes contra la humanidad, señalando que este enfoque es consecuente con el desarrollo de esta categoría de crímenes en Derecho internacional.

En virtud de todo lo expuesto, podría concluirse que las empresas transnacionales serían entidades susceptibles de reunir los criterios formulados por la CPI en su jurisprudencia e integrarse en el alcance de la definición del término organización del art. 7.2 del Estatuto de Roma. Por lo tanto, podría abrirse una vía para que los actos contemplados en el párrafo 1 del art. 7, cuando fueran cometidos por empresas transnacionales, más allá de cualquier colaboración con un

28 El TPIR se pronuncia en este sentido con relación al Apartheid. Causa Akayesu, TC Judgment, párr. 581; causa Rutaganda, TC Judgment, para. 70; caso Musena, TC Judgment, párr. 205.

29 Causa Kunarac de 2001, párr. 421.

30 Comisión de Derecho Internacional (CDI). 2019. Informe de la Comisión de Derecho Internacional. 71er período de sesiones (29 de abril a 7 de junio y 8 de julio a 9 de agosto de 2019). Capítulo IV Crímenes de lesa humanidad. Nueva York: Naciones Unidas: UN Doc. (A/74/10), pp. 10-154. 
tercer actor estatal o con características estatales, dentro del contexto de gravedad para los crímenes, que es su elemento internacional, y que consiste en el hecho de formar parte de un ataque generalizado y sistemático contra la población civil de acuerdo a la política de esa organización, podrían ser susceptibles de calificarse como crímenes contra la humanidad.

El hecho de no reconocer la capacidad que tienen otros actores distintos a los Estados para cometer estos crímenes, impidiendo hacerles penalmente responsables de sus políticas criminales de acuerdo a las normas y principios recogidos en el Estatuto de Roma, significaría no dimensionar ni tratar adecuadamente en el Derecho internacional la grave naturaleza y las importantes consecuencias de las nuevas amenazas que en la sociedad contemporánea se presentan para la humanidad (Kremnitzer 2010).

\section{La competencia de la Corte Penal Internacional y su extensión ratione personae a las empresas transnacionales}

La CPI tiene competencia material sobre los crímenes contra la humanidad y el Estatuto de Roma recoge los diferentes modelos de responsabilidad por la participación en estos crímenes internacionales, consagrando el principio de responsabilidad penal individual, una vez que el DIP no conoce formas de responsabilidad de las personas jurídicas, asociaciones o grupos.

En virtud del artículo 25, la CPI tendría competencia sobre los individuos que, de acuerdo con el Estatuto, ordenasen, propusieran o indujeran la comisión de ese crimen, fueran cómplices o encubridores o colabores de algún modo en la comisión o la tentativa de comisión del crimen, incluso suministrando los medios para su comisión, y, finalmente, contribuyeran de algún otro modo en la comisión o tentativa de comisión del crimen por un grupo de personas que tuvieran una finalidad común (Scheffer 2016).

Aunque a día de hoy la CPI no ha procesado a ningún individuo acusado de participar en un crimen internacional actuando en nombre y representación de una empresa transnacional, el ex fiscal jefe de la CPI, Luis Moreno-Ocampo (2003, 2 y 4), dio viabilidad legal y mostró su disposición general para investigar a los funcionarios y directores de empresas por su participación en crímenes atroces; en particular, para verificar los nexos entre los asesinatos en la República Democrática del Congo y la explotación de recursos minerales. Cuestión que presenta mayor dificultad legal es la que plantea extender la competencia de la 
CPI sobre las empresas transnacionales en calidad de personas jurídicas cuando participan en la comisión de crímenes contra la humanidad, ya sea cooperando con otros actores, o actuando por sí solas de manera autónoma.

La atribución de responsabilidad penal a las personas jurídicas no es nueva para el Derecho internacional, ni para la CPI. En la Conferencia Diplomática de Plenipotenciarios para el establecimiento de una Corte Penal Internacional, celebrada en Roma en 1998, los delegados asistentes discutieron el texto final presentado por el Comité Preparatorio, el cual contenía en el art. 23 una propuesta para extender la jurisdicción ratione personae de la Corte a las personas jurídicas, habiendo sido rechazada por la mayoría de las delegaciones presentes (Van den Herik 2012, Haigh 2008). La extensión de la jurisdicción de la CPI a las personas jurídicas estuvo también en la agenda de la Conferencia de Kampala de 2010 para la revisión del Estatuto de Roma en 2010, pero recibió finalmente muy poca atención. La no inclusión de ésta bajo el paraguas del Estatuto de Roma constituyó una prueba más de que no existía en Derecho internacional una aceptación general de la responsabilidad de las empresas transnacionales (De Brabandere 2011), retrato de la reticencia mostrada por los Estados para regular su responsabilidad penal en los ordenamientos jurídicos nacionales.

Para responder a esta cuestión, como se verá a continuación, se debe tener en consideración una serie de argumentos que provienen del Derecho internacional público, del sistema de la CPI y del Derecho penal.

\section{a) Argumentos de Derecho internacional público}

El consenso sobre la arquitectura del orden jurídico internacional, que descansa en el axioma de la soberanía del Estado -además de otras preocupaciones que giran en torno a la idea de la culpa o del castigo colectivo- es uno de los pilares que milita en contra de reconocer su responsabilidad penal. Este acuerdo se reflejó en la redacción del Proyecto de artículos sobre responsabilidad del Estado por hechos internacionalmente ilícitos, aprobado en primera lectura por la CDI (2001), en el que el término "crímenes internacionales» - recogido en el art. 19 del Proyecto de la CDI (1996) — fue sustituido por la expresión «violaciones graves de obligaciones emanadas de normas imperativas del Derecho internacional general», afirmando así que la responsabilidad del Estado es internacional —ni penal, ni administrativa, ni civil-. 
Ahora bien, la negativa a construir el concepto de la responsabilidad internacional penal de los Estados en Derecho internacional no debería constituir un impedimento para reflexionar sobre el concepto de la responsabilidad penal de las empresas bajo esta rama del Derecho, ya que los Estados y las empresas son actores con distinta subjetividad internacional (Van de Herik y Letnar 2010, Clapham 2013, De Schutter 2006). Si bien los Estados son los actores primigenios y principales del ordenamiento jurídico internacional, en virtud de los principios estructurales de soberanía e igualdad, estos mismos principios no son relevantes para las empresas (Haigh 2008). De este modo, si es difícil imaginar un orden internacional en el que los Estados queden sometidos al ius puniendi de otros Estados, no ocurre lo mismo con las corporaciones. Bajo esta exégesis es posible afirmar que el reconocimiento de la responsabilidad penal de las empresas en Derecho internacional no significaría equiparlas a los Estados en materia de subjetividad.

De esta manera, en Derecho internacional se han venido produciendo algunos avances en materia de reconocimiento de la responsabilidad de las empresas, incluida la responsabilidad penal, por violación de sus normas. La CDI, en su Proyecto de código de crímenes contra la humanidad, adoptado en su 71. a sesión en 2019, decidió incluir en el art. 6, párrafo 8, una disposición sobre la responsabilidad de las personas jurídicas por estos crímenes, tomando en consideración la participación de éstas en actos cometidos como parte de una ataque generalizado o sistemático contra la población civil. Utiliza, sin embargo, una fórmula típica en otros tratados internacionales mediante la que se crea la obligación de los Estados de adoptar todas las medidas necesarias en su legislación nacional, cuando proceda, para hacer responsables a las empresas penalmente -aunque también contempla la responsabilidad civil o administrativa- Asimismo, el borrador revisado del Tratado sobre empresas y derechos humanos, presentado ante el Consejo de Derechos Humanos en julio de 2019 por el Grupo Intergubernamental de Composición abierta para elaborar un instrumento internacional jurídicamente vinculante sobre las empresas transnacionales y otras empresas comerciales en materia de derechos humanos, establece la obligación de las Estados de adoptar mecanismos legales para reconocer la responsabilidad de las empresas por violaciones de derechos humanos en el art. 6. Además, introduce en su párrafo 7 una lista detallada de los crímenes en relación con los que los Estados deben garantizar mecanismos de responsabilidad penal para las empresas, mencionado entre ellos los crímenes contra la 
humanidad ${ }^{31}$. Los «Principios Rectores sobre las Empresas y los Derechos Humanos de las Naciones Unidas »32, que fueron elaborados por el Representante Especial del Secretario General para la cuestión de los derechos humanos y las empresas transnacionales y otras empresas, y hecho suyos por el Consejo de Derechos Humanos en su resolución 17/4, de 16 de junio de 2011, es un precedente de enorme trascendencia en este campo, y también invita a los Estados a adoptar medidas en sus ordenamientos internos para regular la responsabilidad de las empresas. En último lugar, cabe destacar el Protocolo de Malabo, sobre enmiendas al Protocolo del Estatuto de la Corte Africana de Justicia y Derechos Humanos, adoptado en junio de 2014 que, aunque aún no se encuentra en vigor, reconoce la responsabilidad de las empresas por la violación de sus normas (art. 46.c) y la jurisdicción de la Corte sobre las personas jurídicas.

Asimismo, en la dirección de reconocer la responsabilidad penal de las empresas por ilícitos internacionales — transnacionalespueden identificarse significativos esfuerzos provenientes de organizaciones internacionales, como las Naciones Unidas, el Consejo de Europa y la Unión Europea ${ }^{33}$, que empujan a abrazar la noción de la responsabilidad penal de las empresas, incluso entre los Estados continentales que no tienen tradición al respecto (Weigend 2008, 928).

Estas contribuciones y avances significativos en el Derecho internacional son una clara manifestación de la necesidad de abordar el papel de las empresas en el nuevo orden internacional y las realidades del implacable proceso de la globalización (Stessens 2008 , 495) entre las que se observan la comisión de graves crímenes consecuencia de la política corporativa inherente al desarrollo de las actividades económicas de empresas transnacionales.

$31 \mathrm{CDH}$, Borrador revisado por la Presidencia del OEIGWG.16.7.2019.

$32 \mathrm{CDH}$, Principios Rectores sobre las empresas y los derechos humanos, adoptados por el Consejo de Derechos Humanos en su Resolución 17/4, de 16 de junio de 2011.

33 Council of Europe Recommendation R(88) 18, of the Committee of Ministers of Member States concerning liability of enterprises having legal personality for offences committed in the exercise of their activities, 20 October 1988; OECD Convention on Combating Bribery of Foreign Public Officials in International Business Transactions; Special FTAF Recommendation on Terrorist Financing of 22 October 2004, in particular Recommendation 6. Directive 2008/99/EC of the European Parliament and of the Council of 19 November 2008 on the protection of the environment through criminal law, Official Journal L 328, 6 December 2008: 28-37. 
b) Argumentos que derivan del sistema del CPI

La CPI no tiene competencia sobre las personas jurídicas. Las actas oficiales de la Conferencia Diplomática de Plenipotenciarios de las Naciones Unidas para la creación de la CPI muestran que había una división de perspectiva sobre la cuestión, teniendo en consideración las diferencias estructurales entre los diferentes sistemas legales de los Estados participantes, incluyendo la ausencia de regulación de la responsabilidad penal de las personas jurídicas en sus ordenamientos jurídicos ${ }^{34}$.

Si bien Van den Herik $(2012,12)$ señala, a efectos de la extensión de la competencia de la CPI sobre las empresas, que el solo hecho de que haya un gran número de Estados que reconocen su responsabilidad penal proveería una base suficiente para considerar su inclusión en el Estatuto de Roma, Kyriakakis (2008, 122-127) propone que esta labor se emprenda mediante una enmienda al art. 25 del Estatuto de Roma. Este sería un camino lleno de obstáculos políticos (esta cuestión no se encuentra presente en la agenda de una posible reforma al Estatuto de Roma) y de dificultades procedimentales ${ }^{35}$, aunque se debe decir que no son totalmente insalvables.

Ahora bien, aunque el Estatuto de Roma recogiese la extensión de la competencia de la CPI sobre las empresas, se ha puesto de manifiesto el riesgo que esto conllevaría en materia de aplicación del principio de complementariedad de la jurisdicción de la CPI con relación a los foros nacionales (Ambos 2008, 746; Scheffer y Kaeb 2011, 360).

El principio de complementariedad de la CPI se encuentra recogido en el Preámbulo y en el art. 17 del Estatuto de Roma. La falta de regulación del concepto de la responsabilidad penal de las empresas, en un amplio número de jurisdicciones nacionales de los Estados partes, ha venido a sugerir que la ampliación de la jurisdicción ratione personae hacia las empresas en el Estatuto podría traer inconmensurables problemas con relación a este principio (Schabas

34 UN Doc. A/CONF.183/C.1/WGGP/L.5/Rev.2, 3 July 1998.

35 Desde el punto de la revisión del instrumento, las enmiendas al Estatuto de Roma pueden ser propuestas, adoptadas y ratificadas de acuerdo a los art. 121 y 122 del Estatuto. Si bien cualquier Estado puede proponer una enmienda, ésta debe ser adoptada por dos tercios de los votos en la Asamblea de Estados Partes o en conferencia de revisión del Estatuto. Las enmiendas además entrarán en vigor para todos los Estados Partes un año después de ser ratificada por los 7/8 de los Estados Partes. Sin embargo, las enmiendas que afectan a los art. 5, 6, 7 y 8 solo entrarán en vigor para los Estados Partes que ha ratificado la enmienda. 
2008a; Ambos 2008, 746). De hecho, con relación a este principio, el Estatuto refleja el compromiso diplomático de reconciliar dos valores diferentes: por un lado, la soberanía de los Estados, siendo uno de sus rasgos fundamentales el ejercicio de la jurisdicción penal; y, de otro lado, el desarrollo efectivo de la justicia penal internacional (Benzing 2003; Kyriakakis 2008, 123).

La pregunta que surge de fondo es cómo afecta la falta de regulación de la responsabilidad penal de las empresas en las legislaciones nacionales a efectos de la efectividad del principio de complementariedad en el Estatuto de Roma. Kyriakakis (2008) sugiere que este problema relativo a aplicación del principio de complementariedad podría desenmarañarse mediante la misma aplicación del art. 17 del Estatuto de Roma teniendo en consideración las causas de admisibilidad, entre las que se encuentran las siguientes: la incapacidad para investigar o enjuiciar un asunto determinado debido al colapso total o sustancial de la administración nacional de justicia o al hecho de carecer de ella; la imposibilidad de hacer comparecer al acusado, porque no dispone de las pruebas y de los testimonios necesarios; o porque no está «por otras razones» en condiciones de llevar a cabo el juicio, pudiendo tener cabida entre estas "otras razones» la ausencia de legislación adecuada al respecto.

Cassese $(2003,352)$ argumenta que la incapacidad de actuar incluye casos donde los tribunales nacionales no pueden juzgar a una persona, no porque se haya producido un colapso o mal funcionamiento del sistema judicial, sino a causa de impedimentos legales, tales como leyes de amnistía o inmunidades, haciendo imposible para la justicia doméstica comenzar un proceso contra el sospechoso o acusado. Esta lectura sobre la incapacidad de la norma parece incluir la imposibilidad de iniciar procedimientos sobre la base de la ausencia de competencia legislativa sobre la categoría del demandando (Kyriakakis 2008). Siguiendo a reconocidas autoridades en la materia, podría sostenerse que el déficit del Estado en materia de legislación penal sobre las empresas sería un argumento relativamente fácil de presentar en favor de la aceptación de la fórmula sobre la imposibilidad del Estado de perseguir los crímenes establecidos en el Estatuto (Cassese 2003, McCormack y Robertson 2009).

En este sentido, sería posible afirmar que las normas relacionadas con la complementariedad podrían mantenerse sin ser modificadas y el principio de ejercicio de jurisdicción de la CPI podría continuar operando efectivamente. 


\section{c) Argumentos de Derecho penal}

Los tres principales elementos teóricos del Derecho penal que se plantean frente al concepto de la responsabilidad penal de las empresas son que las corporaciones son "ficciones legales», que no pueden actuar independientemente de los individuos que la integran, que no poseen mens rea y que no pueden sufrir una sanción penal (Chiomenti 2006, 292-293). Todos estos argumentos han sido capturados en la expresión «no body to kick, no soul to damn» (Coffee 1981). Esto no significa, sin embargo, que no puedan encontrarse razones de base legal que permitan rebatir estos obstáculos.

En primer lugar, la cuestión de que las empresas no pueden actuar criminalmente de forma autónoma o independiente de los individuos que forman parte de ella, puede ser objetada. Swart $(2008,951)$ señala, por ejemplo, que una vez una interpretación más sociológica de la comisión del crimen ha prevalecido sobre una exégesis más naturalista, no es difícil argumentar que, aunque las empresas no pueden cometer crímenes en el sentido físico de la expresión, sí deciden sobre las prácticas y los procedimientos que las provocan. En este sentido, puede apelarse al «hecho propio» de la empresa a partir de doctrinas tales como corporation policy o management failure (Silva 2016, 300-302).

En segundo lugar, con relación a la construcción del elemento mental —el conocimiento y la intención de actuar - y su conexión con la cuestión de la culpabilidad, puede decirse que de los hechos de las personas jurídicas no puede predicarse una culpabilidad en sentido clásico (Pérez Manzano 1999, 21). Sin embargo, la doctrina ha tratado de eludir estos problemas que presenta la teoría clásica del delito a través de la reconfiguración general del concepto de culpabilidad, la ampliación del concepto de culpabilidad de modo específico para la empresa -el llamado collective knowledge, la culpabilidad por defecto de organización o due diligenceo mediante la elaboración de un concepto paralelo al de la culpabilidad —el del estado de necesidad para los bienes jurídicos(Silva 2016, 311).

El concepto de cultura corporativa puede ayudar también a resolver el conflictivo discurso de que las empresas no pueden actuar moralmente. Las empresas pueden dirigir, fomentar, tolerar o permitir, por acción u omisión, la comisión del crimen (Robinson 2008). Las empresas además son capaces de expresar convicciones morales y legales propias en espacios públicos e influir en la construcción de las políticas públicas y del pensamiento social. Todo 
ello hace que las empresas puedan desarrollar su propia identidad social y su propia personalidad. En este sentido, se entiende que las empresas son actores sociales, con una identidad social propia, y no puede considerarse absurdo desde el punto de vista del Derecho internacional que las empresas puedan ser culpables en una forma distinta a como lo son los seres humanos (Swart 2008, 951-952).

En tercer lugar, para terminar esta cuestión, si aceptamos las ideas de la culpa y el castigo como construcciones sociales, la decisión de aceptar o no la responsabilidad criminal de las corporaciones se presenta como una decisión política, que se puede realizar sobre un análisis de la realidad de los riesgos y las amenazas sociales que provocan, más que una decisión solamente inspirada por consideraciones ontológicas (Chiomenti 2006, 293).

No hacer responsable penalmente a la empresa de un programa o de una política criminal propia significaría no dimensionar, ni tratar adecuadamente, la gravedad de la naturaleza y de las consecuencias de estos comportamientos corporativos (Kremnitzer 2010, 915). Si las conductas criminales de las empresas a las que nos venimos refiriendo, así como sus consecuencias, son especialmente peligrosas y graves es porque éstas forman parte de la cultura corporativa, de las políticas, de los objetivos y de los procedimientos de una empresa, sin cuya arquitectura las personas individuales concretas que forman parte de su estructura organizativa no hubieran procedido u obrado (Geneuss et. al. 2010, 974-975). Éste es un argumento de peso para entender la necesidad político-criminal de atribuir a las empresas responsabilidad directa, y no solo responsabilidad subsidiaria para el caso que no pueda sancionarse a las personas físicas (Kaeb 2016, Van der Wilt 2013).

Uno de los argumentos utilizados de manera reiterativa en favor de la atribución de la responsabilidad penal de las personas jurídicas es que la división del trabajo, que es característica de éstas, produce una importante disolución de la responsabilidad penal individual, surgiendo barreras insuperables para su atribución (Silva 2016, 287). Estas circunstancias dificultan el seguimiento de los procesos judiciales por este tipo de crímenes corporativos (Geneuss et.al. 2010, 974-975) y pueden obstaculizar, o incluso impedir, la reparación adecuada para las víctimas por todos los daños sufridos, incluso dar lugar a la construcción de alternativas a la responsabilidad de la propia persona jurídica al margen del Derecho y del propio proceso penal (Silva 2013, 18).

La sola responsabilidad penal individual puede dar lugar a insuficiencia preventiva. Las condenas dirigidas a empleados, administradores, directivos o representantes dejaran intacta la 
capacidad legal de la empresa, así como de su estructura financiera y operativa, que promueve la comisión del crimen. Además, la ausencia de penas para las empresas - que no sanciones administrativas- por lo simbólico del significado de la intervención del Derecho penal, y la fuerza expresiva y el significado comunicativo de sus consecuencias jurídicas contribuye, sin lugar a dudas, a la construcción de una esfera de cierta impunidad para la actuación de las personas jurídicas (Silva 2013).

En virtud de lo expuesto hasta el momento, desde una perspectiva de política criminal, es inevitable poner de relieve la necesidad de tener un régimen de responsabilidad penal en Derecho internacional para las empresas con el objeto de prevenir, perseguir y sancionar comportamientos criminales que constituyen riegos y amenazas para la protección de aquellos valores humanos básicos reconocidos por la comunidad internacional en su conjunto, cuya protección se encuentra en el núcleo de la prohibición de cometer crímenes contra la humanidad, una vez que el régimen de la responsabilidad penal individual, que es pieza angular en el Estatuto de Roma, se puede mostrar insuficiente o ineficaz para abordar el tipo de criminalidad que se genera en la actividad de la empresa (Silva 2016, 285).

Extender la competencia ratione personae de la CPI a las empresas transnacionales, único órgano judicial internacional penal de carácter permanente con jurisdicción sobre los más graves crímenes de trascendencia internacional, significaría un avance importante en su propósito de prevenirlos y castigarlos (Haigh 2008). Al mismo tiempo, reflejaría un importante progreso a la hora de abordar los enormes desafíos inherentes al desarrollo y a la expansión del Derecho internacional, particularmente del DIP, que debe responder a las rápidas y complejas transformaciones que ha experimentado la sociedad internacional en los últimos tiempos, entre las que se encuentra la participación y el impacto de las empresas transnacionales en su funcionamiento y orden.

\section{Conclusiones}

Las empresas transnacionales pueden estar involucradas en la comisión de crímenes contra la humanidad a partir de diferentes formas de participación, siendo las más frecuentes la complicidad con actores estatales (art. 25.3.c del Estatuto de Roma) y la «empresa criminal conjunta» (art. 25.3.d del Estatuto de Roma). Ahora bien, a día de hoy no se ha abordado con profundidad otras formas de 
participación de las empresas en la comisión de estos aberrantes crímenes más directa y autónoma de terceros. Con ello se quiere poner la atención en si es relevante para la calificación de un crimen contra la humanidad la naturaleza de los actores que se encuentran detrás de la política de cometer estos crímenes, es decir, si el tipo de organización detrás del crimen es un elemento legal o no para su calificación jurídica ${ }^{36}$.

El análisis del concepto «organización» en la jurisprudencia de la CPI nos lleva a pensar que es posible realizar una interpretación más amplia y flexible de esta expresión y que entidades privadas puedan quedar integradas en el ámbito del art. 7 del Estatuto de Roma. De esta manera, las empresas transnacionales son entidades que podrían reunir los criterios recogidos en el «test de capacidad» validado por la CPI, no en todos los contextos ni circunstancias, ya que esta es una cuestión que debería analizarse caso por caso, valorando la concurrencia del contexto de gravedad en el que se comenten las conductas criminales, es decir, el de llevar a cabo un ataque generalizado y sistemático contra la población de conformidad con la política de una empresa transnacional de llevar a cabo ese ataque o promover esa política. Ataque, por otro lado, que se podría concretar en la comisión múltiple de actos de asesinato, desplazamientos forzados, esclavitud, torturas, entre otros, llevados a cabo como medio objetivo, o como resultado o consecuencia, de la actividad económica propia de la empresa. Es decir, crímenes cometidos bien intencionalmente o bien a sabiendas de cuál sería el resultado, aunque sus objetivos fueran otros distintos.

La ampliación de la competencia de la CPI sobre las empresas transnacionales no es una tarea totalmente imposible desde el punto de vista legal. De acuerdo con la opinión de autoridades en la materia, ni los argumentos que derivan del Derecho internacional público, ni del régimen de la competencia complementaria de la CPI, ni de las objeciones teóricas del Derecho penal son obstáculos insuperables para abordar esta tarea (Van den Herik 2012). La creación de un concepto de responsabilidad penal internacional para las empresas por la comisión de crímenes internacionales es necesario desde una perspectiva política criminal. Con ello se pretende demostrar que la magnitud de la naturaleza y de las consecuencias de los crímenes corporativos y su conexión con la realización de crímenes internacionales se establece

36 La CPI ha entrado a investigar los actos de violencia sexual cometidos por organizaciones terroristas como Boko Haram e ISIS y su calificación como crímenes contra la humanidad o crímenes de guerra. Véase International Criminal Court, Office of the Prosecutor, 2017, p. 46 
en el hecho de que los actos criminales forman parte de la cultura, las políticas, los objetivos y los procedimientos de la empresa, sin cuya arquitectura la persona natural ejecutora no hubiera procedido $u$ obrado. Como ha argumentado la Sala de Apelación del TEL «holding only natural persons within a corporation accountable and shielding the corporate entity, as a legal person, from the contempt power of the tribunal would potentially lead to unacceptable impunity for criminal actions and be contrary to the interests of justice» ${ }^{37}$.

Ahora bien, también es necesario señalar que la atribución de la responsabilidad penal internacional a las empresas por la comisión de crímenes contra la humanidad debe ser complementaria la responsabilidad que concierne a las personas físicas que actúan como agentes u órganos bajo la dirección, el control, o como colaboradores de las empresas (Van del Wilt 2013, Kaeb 2016). La inclusión de las organizaciones que son las empresas transnacionales en el ámbito de competencia de la CPI no debe cambiar el enfoque de la responsabilidad penal del individuo, al igual que es posible establecer la responsabilidad internacional del Estado y la responsabilidad penal internacional de sus agentes.

Para concluir solo resta decir que, si bien el DIP no puede considerarse el único remedio para resolver todos los problemas teóricos y prácticos relativos a la participación de las empresas transnacionales en los más graves crímenes internacionales, el Estatuto de Roma y la CPI pueden contribuir a la labor de exigir su responsabilidad penal internacional por la implicación en ellos dotando de las herramientas y conceptos necesarios para ello. La ampliación de su ratione personae a las empresas transnacionales ayudaría a tratar adecuadamente la gravedad y la trascendencia de los crímenes corporativos y su relación con los crímenes internacionales, a acabar con la cultura de impunidad y hacer justicia a las víctimas.

\section{Bibliografía}

Alija, Rosa A. 2011. La persecución como crimen contra la humanidad, Barcelona: UB.

Ambos, Kai. 2008. "Note on article 25», Commentary on the Rome Statute of the International Criminal Court: Observer's Notes, editado por Otto Trifferer, 743-770, Oxford: Hart Publishing.

37 STL, Al Khayat Interlocutory Appeal Concerning Personal Jurisdiction. 
Bartels, Rogier y Katharine Fortin. 2016. «Law, justice and a potential security gap: the 'organization' requirement in International Humanitarian Law and International Criminal law», Journal of Conflict and Security Law 21, n. 1: $1-20$.

Bassiouni, Cherif. 2011. Crimes against humanity. Historical evolution and contemporary application, Nueva York: Cambridge University Press.

Benzing, Markus. 2003. "The complementarity regime of the International Criminal Court: international criminal justice between state sovereignty and the fight against impunity». Max Planck Yearbook of United Nations Law 7.

Bonet, Jordi. 2013. El derecho Internacional de los derechos humanos en periodos de crisis. Madrid: Marcial Pons.

Cassese, Antonio. 2003. International Criminal Law, Oxford: Oxford University Press.

Chiomenti, Cristina. 2006. "Corporations and the International Criminal Court», en Transnational Corporations and Human Rights, editado por Olivier De Schutter, 287-312, Oxford: Hart Publishing.

Clapham, Andrew. 2013. Human Rights Obligations of Non-States Actors, Oxford: Oxford University Press.

Coffee, J. C. 1981. "No soul to damn: no body to kick": an unscandalized inquiry into the problem of corporate punishment». Michigan Law Review 79, n. 3: 386-459.

Comisión de Derecho Internacional (CDI). 1996. Yearbook of the International Law Commission, vol. II, Second Part. Report of the Commission to the General Assembly on the work of its forty-eighth session. UN Doc. A/CN.4/ SER.A11996/Add.I, Part 2.

Comisión de Derecho Internacional (CDI). 2001. Anuario de la Comisión de Derecho Internacional. Vol. II, Parte 2. Informe de la Comisión a la Asamblea General sobre la labor realizada en su quincuagésimo tercer período de sesiones. A/CN.4/SER.A/2001/Add.1, Part 2.

Comisión de Derecho Internacional (CDI). 2019. Informe de la Comisión de Derecho Internacional 71er período de sesiones (29 de abril a 7 de junio y 8 de julio a 9 de agosto de 2019). Nueva York: Naciones Unidas: 10-154.

De Brabandere, Eric. 2011. "Non-state actors and human rights: corporate responsibility and the attempts to formalize the role of corporations as participants in the international legal system» en Participants in the International Legal System. Multiple Perspectives on Non-State Actors in International Law, editado por Jean D'Aspremont, 268-283, Abingdon: Routledge.

De Schutter, Olivier. 2006. Transnational Corporations and Human Rights, Studies in International Law. Oxford: Hart Publishing.

Di Fillipo, Marcello. 2008. «Terrorist crimes and international co-operation: critical remarks on the definition and inclusion of terrorism in the category of international crimes», European Journal of International Law 19: 533-570.

Donigan, Carrie-Lyn. 2000. «International legal mechanisms for combating organized crime: the need for a multilateral convention». Berkeley Journal of International Law 18, n, 1: 53-101. 
Fraser, David. 2017. «(De)constructing the nazi State: criminal organizations and the Constitutional Theory of the International Military Tribunal», Loyola of Los Angeles International and Comparative Law Review 39: 117186.

Geneuss, Julia, Jan P. Book, Boris Burghardt y Oliver Schüttpelz. 2010. "Core crimes Inc. Panel discussion reports from the Conference on "Transnational Business and International Criminal Law"», Journal of International Criminal Justice 8: 957-977.

Haigh, Kathryn. 2008. «Extending the International Criminal Court's jurisdiction to corporations: overcoming complementarity concerns» Australian Journal of Human Rights 14, n. 1: 199-219.

Jørgensen, Nina. 2001. "A reappraisal of the abandoned Nuremberg concept of criminal organisations in the context of justice in Rwanda», Criminal Law Forum 12, n. 3: 371-406.

Kaeb, Caroline. 2016. "The shifting sands of corporate liability under international criminal law». The George Washington International Law Review 49: 351-403.

Kaleck, Wolfgang y Miriam Saage-MaaB. 2010. "Corporate accountability for human rights violations amounting to international crimes the status quo and its challenges». Journal of International Criminal Justice 8: 699-724.

Kremnitzer, Markus. 2010. «A possible case for imposing criminal liability on corporations in International Criminal Law», Journal of International Criminal Justice 8, n. 3: 909-918.

Kyriakakis, Joanna. 2008. "Corporations and the International Criminal Court: the complementarity principle stripped bare», Criminal Law Forum 19, n. 1: 115-151.

Márquez, Carmen. 2008a. El proceso de codificación y desarrollo progresivo de los crímenes contra la humanidad. Sevilla: Universidad de Sevilla.

Márquez, Carmen. 2008b. «Los elementos comunes de los crímenes de lesa la humanidad», Revista General de Derecho Penal 9: 1-56.

McAuliffe de Guzman, Margaret. 2000. «The road from Rome: The developing law of crimes against Humanity». Human Rights Quarterly 22: 335-403.

McCormack, Timothy y Susan Robertson. 2009. "Jurisdictional aspects of the Rome Statute for the new International Criminal Court», Melbourne University Law Review 23. Acceso el 25 de marzo de 2020. http://classic. austlii.edu.au/au/journals/MelbULawRw/1999/25.html

Moran, Clare F. 2014. "Human trafficking and the Rome statute of the international criminal court», The Age of Human Rights Journal 3: 32-45.

Moreno-Ocampo, Luis. 2003. Second Assembly of State Parties to the Rome Statute of the International Criminal Court, Report of the Prosecutor of the ICC. La Haya; Internacional Criminal Court.

Naucke, Wolfgang. 2015. El concepto de delito económico-político. Una aproximación, Madrid: Marcial Pons.

Oboka, Tomoya. 2005. "Trafficking of human beings as a crime against humanity: some implications for the international legal system», International and Comparative Law Quarterly 54, n. 2: 445-457. 
Pérez Caballero, Jesús. 2015. El elemento político en los crímenes contra la humanidad. La expansión de la figura al crimen organizado transnacional $y$ el caso de las organizaciones de narcotraficantes mexicanas en el sexenio 2006-2012, Madrid: Dykinson.

Pérez Manzano, Mercedes. 1999. Autoría y participación imprudente en el Código Penal de 1995, Madrid: Civitas.

Robinson, Allens A. 2008. Corporate Culture' As A Basis for The Criminal Liability Of Corporations, prepared for the United Nations Special Representative of the Secretary General on Human Rights and Business. Acceso el 30 de marzo de 2020. https://media.business-humanrights.org/ media/documents/f72634fd87adfd3d31a22f5f4b93150267b8a764.pdf

Robinson, Darryl. 2011. «Essence of crimes against humanity raised by challenges at ICC», Blog of the European Journal of International Law, EJIL Talk!, 27 septiembre.

Sadat, Leyla. 2013. "Crimes against Humanity in the Modern Age». The American Journal of International Law 107, n. 334: 1-94.

Schabas, William. 2008a. "General principles of criminal law in the International Criminal Court (Part III)». European Journal of Crime, Criminal Law and Criminal Justice 6, n. 4: 400-428.

Schabas, William. 2008b. "State policy as an element of international crimes», Journal of Criminal Law and Criminology 98, n. 3: 953-982.

Scheffer, David. 2016. "Corporate liability under the Rome Statute». Harvard International Law Journal 57: 35-39.

Scheffer, David y Caroline Kaeb. 2011. "The five levels of CSR compliance: the resiliency of corporate liability under the Alien Tort Statute and the case for a counterattack strategy in compliance theory» Berkeley Journal of International Law 29: 334-397.

Silva, Jesús M. (dir.). 2013. Criminalidad de la empresa y compliance. Prevención y reacciones corporativas, Barcelona: Atelier Libros Jurídicos.

Silva, Jesús M. 2016. Fundamentos del Derecho penal de la Empresa, Madrid: Edisofer.

Smith, Jennifer. 2009. "An international hit job: prosecuting organized crime acts as crimes against humanity», The Georgetown Law Journal 97: 1111-1154.

Stessens, Guy. 2008. "Corporate criminal liability: a comparative perspective». International and Comparative Law Quarterly 43, n. 3: 493-520.

Swart, Ben. 2008. «Discussion. International trends towards establishing some form of punishment for corporations» Journal of International Criminal Justice 6, n. 5: 947-979.

UN (1998). United Nations Diplomatic Conference of Plenipotentiaries on the Establishment of an International Criminal Court, 15 June - 17 July.

Van den Herik, Larissa. 2012 «Subjecting corporations to the ICC regime: analyzing the legal counterarguments». The review conference and the future of the International Criminal Court, 22 Enero.

Van den Herik, Larissa y Jernej Letnar. 2010. «Regulating corporations under international law. From Human Rights to International Criminal Law and back again». Journal of International Criminal Justice 8, n. 3: 725-743. 
Van der Wilt, Harmen. 2013. «Corporate criminal responsibility for international crimes: exploring the possibilities», Chinese Journal of International Law 12, n. 1: 43-77.

Van der Wilt, Harmen. 2014 Trafficking in human beings: a modern form of slavery or a transnational crime? Research Paper, Amsterdam Center for International Law.

Weigend, Thomas. 2008. "Societas delinquere non potest? A German Perspective». Journal of International Criminal Justice 6, n. 5: 927-945. 


\section{Copyright}

Deusto Journal of Human Rights / Revista Deusto de Derechos Humanos is an Open Access journal; which means that it is free for full and immediate access, reading, search, download, distribution, and reuse in any medium only for non-commercial purposes and in accordance with any applicable copyright legislation, without prior permission from the copyright holder (University of Deusto) or the author; provided the original work and publication source are properly cited (Issue number, year, pages and DOI if applicable) and any changes to the original are clearly indicated. Any other use of its content in any medium or format, now known or developed in the future, requires prior written permission of the copyright holder.

\section{Derechos de autoría}

Deusto Journal of Human Rights / Revista Deusto de Derechos Humanos es una revista de Acceso Abierto; lo que significa que es de libre acceso en su integridad inmediatamente después de la publicación de cada número. Se permite su lectura, la búsqueda, descarga, distribución y reutilización en cualquier tipo de soporte sólo para fines no comerciales y según lo previsto por la ley; sin la previa autorización de la Editorial (Universidad de Deusto) o la persona autora, siempre que la obra original sea debidamente citada (número, año, páginas y DOI si procede) y cualquier cambio en el original esté claramente indicado. Cualquier otro uso de su contenido en cualquier medio o formato, ahora conocido o desarrollado en el futuro, requiere el permiso previo por escrito de la persona titular de los derechos de autoría. 